\title{
富电子杂环芳烃与酮酯的自由基脱氢偶联反应
}

\author{
肖莹霞 ${ }^{a}$ 柳忠全*,,$b$ \\ ( ${ }^{a}$ 兰州大学化学化工学院 功能有机分子化学国家重点实验室 兰州 730000) \\ ( ${ }^{b}$ 南京中医药大学药学院 南京 210023)
}

\begin{abstract}
摘要 报道了一种小分子酮、酯与富电子杂环芳烃的高度选择性的自由基脱氢交叉偶联反应. 酯、酮作为溶剂，过氧 化物加热条件下发生裂解与酯、酮作用产生 $\alpha$ 羰基碳中心自由基，进而与富电子杂环芳烃发生交叉脱氢偶联，得到一 系列 C-2 官能化富电子杂环产物. 该反应成功地运用自由基的极性效应，从而精确控制自由基反应的化学选择性. 此 外，该体系还具有反应迅速、操作简便、官能团兼容性较好、区域选择性单一等优点. 预期它将在合成有机化学上得 到较广泛的应用.
\end{abstract}

关键词 自由基; $\mathrm{C}-\mathrm{H}$ 活化; 酮酯; 交叉脱氢偶联; 富电子杂环芳烃

\section{Radical-Promoted Cross Dehydrogenative Coupling of Ketones and Esters with Electron-Rich Heteroarenes}

\author{
Xiao, Yingxia $^{a} \quad$ Liu, Zhong-Quan* ${ }^{*, a}$ \\ ( ${ }^{a}$ State Key Laboratory of Applied Organic Chemistry, College of Chemistry and Chemical Engineering, Lanzhou University, \\ Lanzhou 730000) \\ ( ${ }^{b}$ College of Pharmacy, Nanjing University of Chinese Medicine, Nanjing 210023)
}

\begin{abstract}
The cross dehydrogenative coupling (CDC) via highly selective $\mathrm{C}-\mathrm{H}$ bond functionalization represents one of the most atom-economical, environmentally-benign and efficient synthetic strategies. For a long time, the cleavage of $\mathrm{C}-\mathrm{H}$ bonds initiated by free radicals has been regarded as unselective and useless. However, more and more studies have shown that free radical mediated strategies could also achieve $\mathrm{C}-\mathrm{H}$ bond functionalization in high selectivity recently. In general, it's well-known that nucleophilic free radical species tend to extract hydrogen atoms on electron-deficient $\mathrm{C}-\mathrm{H}$ bonds, while electrophilic free radicals abstract hydrogen atoms on electron-rich $\mathrm{C}-\mathrm{H}$ bonds. A recent study by our group shows that after thermal decomposition of peroxy tert-butyl ether, the electron-rich methyl radicals are produced. Then the radical cleavage of the $\mathrm{C}\left(\mathrm{sp}^{3}\right)-\mathrm{H}$ bond in ketone/ester would happen prior to the $\alpha$-carbonyl-C $-\mathrm{H}$ bond. Subsequently, the electrophilic $\alpha$-carbonyl-C-centered radical selectively reacted with electron-rich olefins to afford new $\mathrm{C}-\mathrm{C}$ bonds. Here, a free-radical initiated highly selective cross dehydrogenative coupling reaction of simple ketones and esters with electron-rich heteroarenes was demonstrated. The ketones and esters were used as solvent, and they would afford the corresponding $\alpha$-carbonyl C-centered radicals, which then add to heteroaromatics leading to a series of C(2)-functionalized heterocycles. The chemoselectivity of this system was well-controlled by application of the polar effect of free radicals. In addition, this protocol features fast, simple operation, good functional group tolerance and site specific etc. The potential of this method was demonstrated through the synthesis of non-steroidal anti-inflammatory and analgesic drug tolmetin. It is expected to have wide applications in synthetic organic chemistry. Typical reaction conditions are as follows: a mixture of heteroarenes (1 equiv., $0.20 \mathrm{mmol})$, TBPA ( 3 equiv., $0.06 \mathrm{mmol}$ ) and ketones/esters $(6 \mathrm{~mL})$ was heated under reflux at $130{ }^{\circ} \mathrm{C}$ for about $1 \mathrm{~h}$. After completion of the reaction, the crude product was cooled to room temperature, the excess solvent was recovered by rotary evaporator and the residue was further purified by column chromatography on silica gel to obtain the desired product (eluent: petroleum ether/ethyl acetate).
\end{abstract}

Keywords free radical; $\mathrm{C}-\mathrm{H}$ activation; ketone and ester; cross dehydrogenative coupling (CDC); electron-rich heteroarene

\section{1 引言}

高选择性的 $\mathrm{C}-\mathrm{H}$ 键官能团化并实现交叉脱氢偶联
(CDC) 是最原子经济、绿色高效的合成策略之一 ${ }^{[1,2]}$. 长 期以来，自由基引发切断 $\mathrm{C}-\mathrm{H}$ 键被认为是没有选择性 的，是杂乱无章的. 然而近年来，越来越多的研究表明，

*E-mail: liuzhq@1zu.edu.cn; Tel.: 0931-8912500; Fax: 0931-8915557

Received May 21, 2019; published June 21, 2019.

Supporting information for this article is available free of charge via the Internet at http://sioc-journal.cn.

Project supported by the National Natural Science Foundation of China (No. 21672089), the State Key Laboratory of Applied Organic Chemistry of Lanzhou University and the Nanjing University of Chinese Medicine.

项目受国家自然科学基金(No. 21672089)、兰州大学功能有机分子化学国家重点实验室以及南京中医药大学资助. 
自由基化学策略同样也可以实现高选择性的 $\mathrm{C}-\mathrm{H}$ 键官 能团化 ${ }^{[3]}$. 当然, 这需要精确调控自由基的极性 ${ }^{[4]}$. 普遍 的经验规律是：亲核性的自由基物种倾向于提取缺电子 $\mathrm{C}-\mathrm{H}$ 键上的氢原子; 而亲电性的自由基物种则提取富 电子 $\mathrm{C}-\mathrm{H}$ 键上的氢原子. 例如: $\mathrm{Ryu}$ 等 ${ }^{[5]}$ 研究发现, 十 聚钨酸盐, 在光照下, 生成亲电性氧中心自由基, 再提 取酮 $\beta$-位 $\mathrm{C}-\mathrm{H}$, 进而与缺电子烯烃发生 Giese 反应. 我 们课题组 ${ }^{[6]}$ 最近的一项研究表明, 过氧叔丁醚受热分解 后产生富电子的甲基自由基，选择性地提取缺电子的酮 $\alpha$-位 $\mathrm{C}-\mathrm{H}$, 从而得到亲电性的邻羰基碳中心自由基, 接着与富电子烯烃发生原子转移自由基加成. 在过去几 十年里, 通过自由基引发选择性官能团化简单酮中饱和 $\mathrm{C}-\mathrm{H}$ 键的研究取得了一些可喜的进展 ${ }^{[7]}$. 然而, 酮酯类 化合物与富电子杂环芳烃的直接氧化脱氢交叉偶联反 应至今仍未见报道. 在此, 我们报道首例富电子杂环芳 烃与小分子酮、酯的自由基 CDC 反应(Scheme 1).

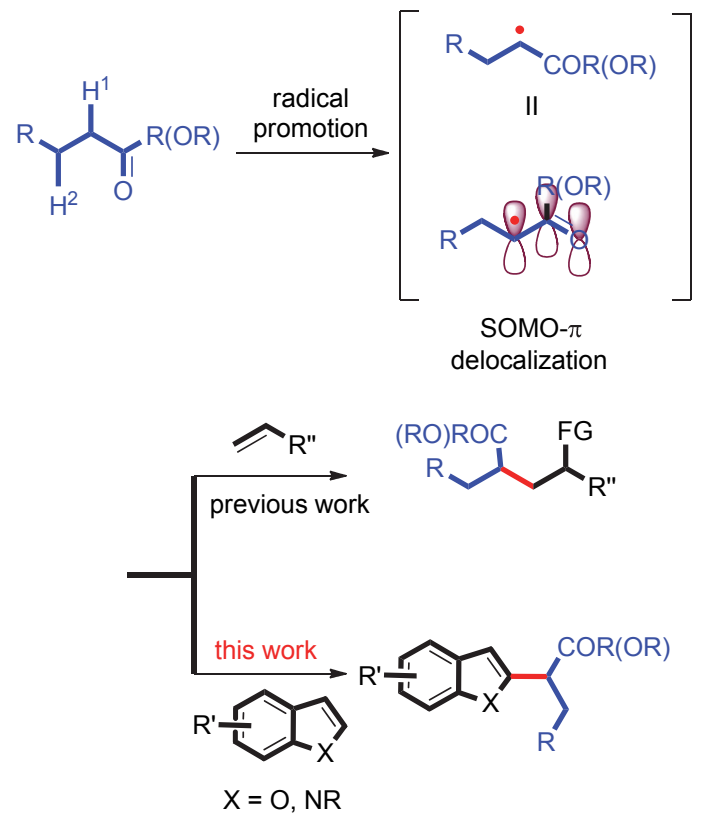

图式 1 自由基促进的酮、酯中 $\mathrm{C}-\mathrm{H}$ 键转化

Scheme 1 Free-radical-promoted conversions of the $\mathrm{C}-\mathrm{H}$ bond in ketone/ester

\section{2 结果与讨论}

\section{1 反应条件的优化}

为了得到最优的反应条件, 选用 $N$-甲基吲哚与醋 酸叔丁酯作为模型分子, 考察了一系列反应条件, 发现 自由基引发剂和温度对反应有着重要的影响(表 1), 在 催化量的乙酰丙酮铁存在下, 叔丁基过氧化氢为自由基 引发剂, $130{ }^{\circ} \mathrm{C}$ 下封管中反应 $12 \mathrm{~h}$, 以 $8 \%$ 的收率得到预 期产物(Entry 1). 改变引发剂种类(过氧叔丁醚、过氧化 异丙苯和过氧化乙酸叔丁酯), 发现产率有所提高 (Entries 2 5). 接着, 在不加入金属催化剂的情况下发
现过氧化乙酸叔丁酯为引发剂能够以 $54 \%$ 的产率得到 目标产物. 接下来考察了溶剂笼效应，当醋酸叔丁酯的 体积为 $6 \mathrm{~mL}$ 时得到产率最高(Entries 8 10). 延长反应 时间和减少引发剂的量都会导致反应产率降低(Entries 11 12). 降低反应温度同样会使反应产物减少. 最终我 们得到最优条件是: 1 equiv. 的 $N$-甲基吲哚, $6 \mathrm{~mL}$ 醋酸叔 丁酯为溶剂, 3 equiv. 的过氧化乙酸叔丁酯位自由基引发 剂, 反应温度为 $130{ }^{\circ} \mathrm{C}$, 以 $60 \%$ 的产率得到反应产物.

表 1 反应条件篮选 ${ }^{a}$

Table 1 Optimization of the reaction

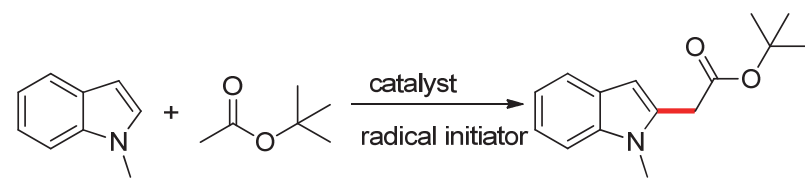

\begin{tabular}{|c|c|c|c|c|c|c|}
\hline Entry & Radical initiator & Peroxide (equiv.) $\mathrm{S}$ & Sol./m & $t / \mathrm{hT}$ & Tem. $/^{\circ} \mathrm{C}$ & Yield $^{b} / \%$ \\
\hline 1 & $\mathrm{Fe}(\mathrm{acac})_{3}(10 \mathrm{~mol} \%)$ & TBHP(decane) (3) & 5 & 12 & 130 & 8 \\
\hline 2 & $\mathrm{Fe}(\mathrm{acac})_{3}(10 \mathrm{~mol} \%)$ & DTBP (3) & 5 & 12 & 130 & 25 \\
\hline 3 & $\mathrm{Fe}(\mathrm{acac})_{3}(10 \mathrm{~mol} \%)$ & $\mathrm{K}_{2} \mathrm{~S}_{2} \mathrm{O}_{8}(3)$ & 5 & 12 & 130 & 0 \\
\hline 4 & $\mathrm{Fe}(\mathrm{acac})_{3}(10 \mathrm{~mol} \%)$ & DCP (3) & 5 & 12 & 130 & 39 \\
\hline 5 & $\mathrm{Fe}(\mathrm{acac})_{3}(10 \mathrm{~mol} \%)$ & TBPA (3) & 5 & 1 & 130 & 38 \\
\hline 6 & - & DCP (3) & 5 & 12 & 130 & 24 \\
\hline 7 & 一 & TВPA (3) & 5 & 1 & 130 & 54 \\
\hline 8 & - & TBPA (3) & 4 & 1 & 130 & 45 \\
\hline 9 & - & TBPA (3) & 6 & 1 & 130 & 60 \\
\hline 10 & - & TBPA (3) & 7 & 1 & 130 & 51 \\
\hline 11 & - & ТВРА (3) & 6 & 2 & 130 & 47 \\
\hline 12 & - & TBPA (2) & 6 & 1 & 130 & 32 \\
\hline 13 & - & TBPA (3) & 6 & 1 & 120 & 26 \\
\hline
\end{tabular}

${ }^{a}$ Reaction conditions: $N$-methyl indole (1 equiv., $0.2 \mathrm{mmol}$ ), tert-butyl acetate as solvent, sealed tube, unless otherwise noted; ${ }^{b}$ Isolated yields.

\section{2 底物适用范围考察}

在最佳反应条件下，考察了一系列富电子杂环底 物, 均以中等到良好的产率得到目标产物(表 2). 芳环上 不同甲基取代的 $N$-甲基吲哚以中等产率得到相应的 $\mathrm{C}(2)$ 官能化产物 $(\mathbf{2} \sim 5)$. 吲哚中 $\mathrm{C}(5)$ 位取代的卤素如氟、 氯、溴和碘在该体系中都能稳定存在，以中等产率发生 单一位置的 $\mathrm{CDC}$ 反应 $(6 \sim 9)$. 紧接着考察了电子效应对 该反应的影响. 富电子的取代基团如甲氧基和苄氧基的 吲哚也能够顺利进行反应 $(\mathbf{1 0}, \mathbf{1 1})$. 令人高兴的是 $C(3)$ 位吸电子基团如羧基、乙酰基和酯基取代的吲哚分别以 76\%, 74\%和 73\%的良好产率得到目标产物 $(12 \sim 14)$. 一 些敏感活泼的官能团如羟基和烷基溴在该条件下均能 耐受 $(15 \sim 17)$. 吡咯类的含氮杂环同样能够进行该类型 反应 $(19 \sim 22)$, 其中值得注意的是, $N$-甲基吡咯得到了 单取代和双取代的混合产物. 不同取代的呋喃类底物在 相应的反应条件下得到 C(2)位取代的相应产物(23 26). 有意思的是, 底物 $\mathrm{C}(3)$ 位取代的呋喃反应位点发生 在 $\mathrm{C}(2)$ 位, 而不是位阻效应更小的 $\mathrm{C}(5)$ 位. 这可能跟反 应生成更稳定的苄位自由基中间体密切相关(24). 接下 
表 2 杂环的底物拓展 ${ }^{a}$

Table 2 Radical CDC reaction of ketones and esters with $N$-heterocycles.

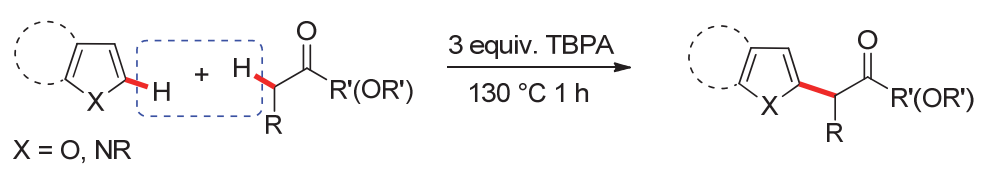

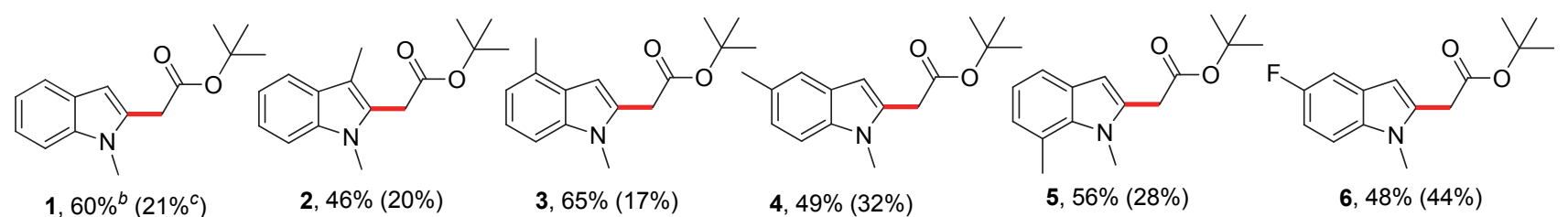

$1,60 \%^{b}\left(21 \%^{c}\right)$

2, $46 \%(20 \%)$

3, $65 \%(17 \%)$

4, $49 \%(32 \%)$

$5,56 \%(28 \%)$

$6,48 \%(44 \%)$<smiles></smiles>
$7,61 \%(29 \%)$ 8, $30 \%(26 \%)$ 9, $54 \%(37 \%)$

$10,42 \%(36 \%)$

$11,59 \%(25 \%)$<smiles>CC(=O)c1c(CC(=O)OC(C)(C)C)n(C)c2ccccc12</smiles>

$12,74 \%(26 \%)$<smiles>Cn1c(CC(=O)OC(C)(C)C)c(C(=O)O)c2ccccc21</smiles>

$13,76 \%(24 \%)$<smiles>COC(=O)Cc1c(C(=O)OC)c2ccccc2n1C</smiles>

$14,73 \%$<smiles>C/C=C\C(=O)OC(C)(C)C</smiles>

$15,83 \%$<smiles>COCCc1c(CC(=O)OC(C)(C)C)n(C)c2ccccc12</smiles>

$16,62 \%$<smiles>CC(C)OC(=O)Cc1c(CCBr)c2ccccc2n1C</smiles>

$17,42 \%(16 \%)$<smiles>CCCCn1c(CC(=O)OC(C)(C)C)ccc1CC(=O)OC(C)(C)C</smiles>

$18,44 \%(33 \%)$

19, $40 \%$

$20,22 \%(24 \%)$

21, $72 \%$

22, $54 \%(36 \%)$<smiles>CC(C)(C)OC(=O)Cc1ccc(-c2ccccc2)o1</smiles>

23, $41 \%(28 \%)$<smiles>COc1ccc(-c2ccoc2CC(=O)OC(C)(C)C)cc1</smiles>

$24,38 \%(18 \%)$<smiles>COc1ccc(-c2ccc(CC(=O)OC(C)(C)C)o2)cc1</smiles>

$25,40 \%(26 \%)$

26, $29 \%(28 \%)$<smiles>COC(=O)Cc1cc2ccccc2n1C</smiles>

27, $75 \%(23 \%)$<smiles>COC(=O)Cc1cc2cc(C#N)ccc2n1C</smiles>

28, $40 \%(21 \%)$<smiles>CC(=O)Cc1ccc(-c2ccccc2)o1</smiles>

34, $42 \%(21 \%)$
$29,46 \%(41 \%)$<smiles>CC(=O)C(C)c1ccc(-c2ccccc2)o1</smiles>

$35,35 \%(25 \%)$<smiles>COC(=O)Cc1cc2ccccc2[nH]1</smiles>

30, $34 \%(35 \%)$<smiles>CCC(=O)C(C)c1ccc(-c2ccccc2)o1</smiles>

36, $44 \%(20 \%)$<smiles>CCOC(=O)C(C)c1cc2ccccc2n1C</smiles>

$31,51 \%(19 \%)$<smiles>CC(C)C(=O)C(C)(C)c1ccc(-c2ccccc2)o1</smiles>

$37,31 \%(30 \%)$<smiles>CCOC(=O)Cc1c(C)c2ccccc2n1C</smiles>

32, $54 \%(17 \%)$<smiles>CC(=O)C(C)(C)c1ccc(-c2ccccc2)o1</smiles>

38, $30 \%$ (36\%)

${ }^{a}$ Reaction condition: heteroarene ( 1 equiv., $\left.0.2 \mathrm{mmol}\right)$, TBPA ( 3 equiv., $\left.0.6 \mathrm{mmol}\right)$, ester/ketone as solvent $(6 \mathrm{~mL}), 130{ }^{\circ} \mathrm{C}, 1 \mathrm{~h}, \mathrm{sealed}$ tube; furan $(1 \mathrm{equiv} ., 0.2$ mmol), CuI $(10 \% \mathrm{~mol}, 0.02 \mathrm{mmol})$, ketones as solvent $(5 \mathrm{~mL}), \mathrm{N}_{2}, 115{ }^{\circ} \mathrm{C}, 12 \mathrm{~h}$, sealed tube, unless otherwise noted. ${ }^{b}$ Isolated yields. ${ }^{c}$ Recovery of starting materials (rsm).

来，考察了一系列低沸点的酯和酮与杂环芳烃的反应情 况. 乙酸甲酯、乙酸乙酯和丙酸乙酯与吲哚的反应高选 择性地发生在羰基 $\alpha-\mathrm{C}\left(\mathrm{sp}^{3}\right)-\mathrm{H}$ 位置 $(27 \sim 32)$. 杂环与各 种酮交叉脱氢偶联反应顺利进行 $(33 \sim 38)$. 不对称的酮
均以高选择性发生在单一位点，这表明反应中自由基中 间体的稳定性起到了决定性的作用.

此外，该反应可以顺利地实现克级生产.例如，如 Scheme 2 所示, 运用该方法作为关键步骤，可以十分有 
效地合成非甾体消炎镇痛药物托美汀. 相比以往大多数 合成方法，该合成策略更绿色环保、更经济安全.

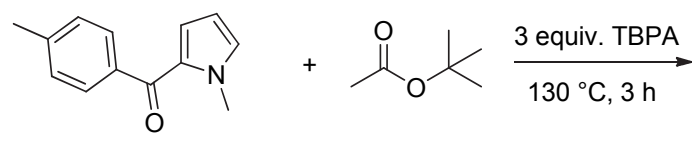

$5 \mathrm{mmol} 1.0 \mathrm{~g}$

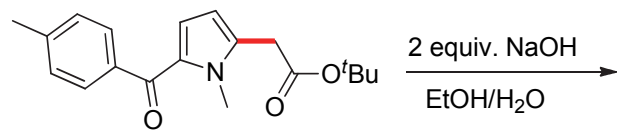

$3.5 \mathrm{mmol} 1.1 \mathrm{~g}$

$70 \%$

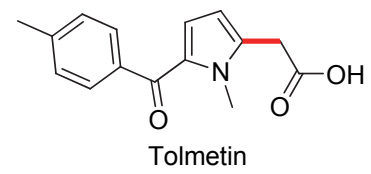

$3.43 \mathrm{mmol} 0.855 \mathrm{~g}$

$95 \%$

图式 2 合成应用

Scheme 2 Synthetic application

\section{3 反应机理推测}

我们进行了一系列实验来研究该反应的机理 (Scheme 3). 首先在该反应体系中加入过量的自由基捕 截剂 2,2,6,6-四甲基哌啶氧化物(TEMPO), 反应被彻底 抑制, 没有观测到预期产物的生成. 接下来进行了两组 平行反应来探索该反应的気代动力学同位素效应(KIE). 在最优条件下以 $N$-甲基吲哚为原料, 分别以丙酮和 $d_{6^{-}}$ 丙酮作溶剂, 得到相应产物比例为 $2: 1$, 表明酮酯分子 中 $\alpha$-羰基位饱和 $\mathrm{C}-\mathrm{H}$ 键的断裂可能包含在该反应的决 速步骤中.
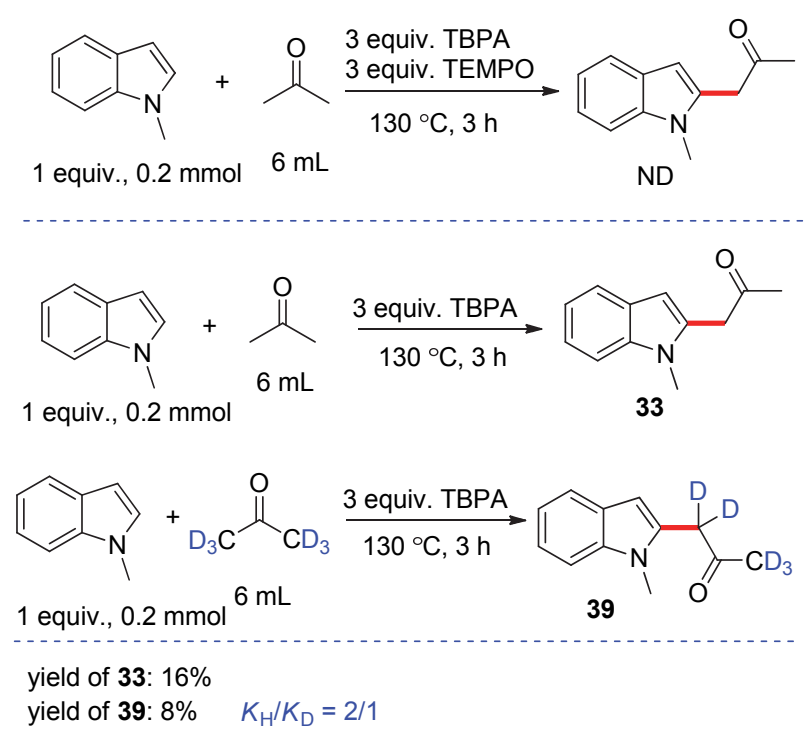

图式 3 机理研究

Scheme 3 Mechanistic studies
根据以上实验数据和我们以往的研究 ${ }^{[8]}$, 提出了以 下可能的反应机理(Scheme 4). 加热条件下，过氧化乙 酸叔丁酯(TBPA)中 $\mathrm{O}-\mathrm{O}$ 键发生均裂, 产生乙酸自由基 和叔丁氧自由基，羧酸自由基脱羧或者烷氧自由基发生 $\beta$ 断裂产生甲基自由基; 亲核性的 $\mathrm{CH}_{3}$ 自由基与酮/酯中 $\alpha$-羰基位 $\mathrm{C}\left(\mathrm{sp}^{3}\right)-\mathrm{H}$ 键发生氢提取, 得到自由基中间体 $\mathbf{A}$, 亲电性的自由基 $\mathbf{A}$ 加成到富电子杂环芳烃得中间体 $\mathbf{B} ， \mathbf{B}$ 与体系中的甲基自由基或乙酸或叔丁氧自由基发 生氢原子提取或者经单电子氧化再脱质子得到最终产 物.

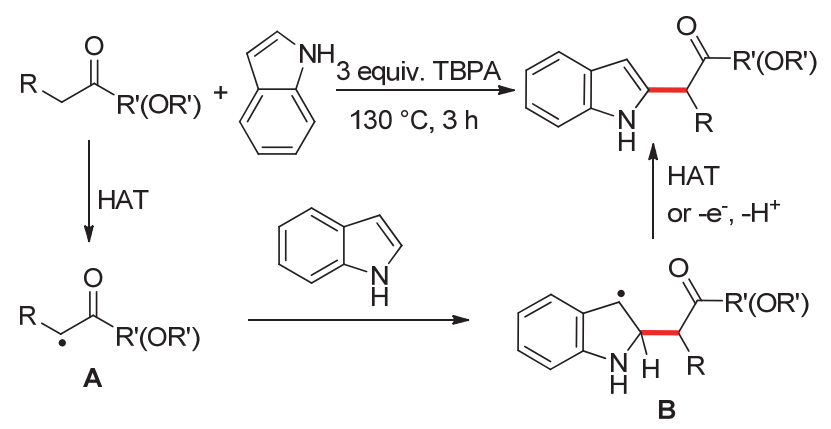

图式 4 推测的反应机理

Scheme 4 Proposed mechanism

\section{3 结论}

综上所述，发展了一种自由基促进的简单的酮、酯 小分子与富电子杂环芳烃的 CDC 反应. 通过该反应可 以绿色高效地制备一系列吲哚、吡咯和呋喃 $\mathrm{C}(2)$ 位置 酮、酯取代的衍生物。同时，该策略有望应用于工业合 成药物分子中. 通过自由基策略实现 $\mathrm{C}\left(\mathrm{sp}^{3}\right)-\mathrm{H}$ 键的高 度选择性转化的研究正在本实验室进行之中.

\section{References}

[1] For selected recent reviews on CDC reactions, see: (a) Li, C.-J. Acc. Chem. Res. 2009, 42, 335. (b) Yeung, C. S.; Dong, V. M. Chem. Rev. 2011, 111, 1215. (c) Girard, S. A.; Knauber, T.; Li, C.-J. Angew. Chem., Int. Ed. 2014, 53, 74. (d) Jia, F.; Li, Z. Org. Chem. Front. 2014, 1, 194. (e) Zhang, J.; Lu, Q.; Liu, C.; Lei, A. Chin. J. Org. Chem. 2015，35，743. (张剑，陆庆全，刘超，雷爱文，有机化学， 2015, 35, 743.) (f) Zhang, G.; Bian, C.; Lei, A. Chin. J. Catal. 2015 36, 1428. (g) Pei, P.; Zhang, F.; Yi, H.; Lei, A. Acta Chim. Sinica 2017, 75, 15. (裴朋昆, 张凡, 易红, 雷爱文, 化学学报, 2017, 75, 15.) (h) Shao, A.; Li, N.; Gao, Y.; Zhan, J.; Chiang, C. W.; Lei, A. Chin. J. Chem. 2018, 36, 619; (i) Liu, Y.; Yi, H.; Lei, A. Chin. J. Chem. 2018, 36, 692.

[2] For selected recent reviews on $\mathrm{C}-\mathrm{H}$ functionalization, see: (a) Zhang, S.; Zhang, F.; Tu, Y.-Q. Chem. Soc. Rev. 2011, 40, 1937. (b) Davies, H. M. L.; Morton, D. Chem. Soc. Rev. 2011, 40, 1857. (c) Newhouse, T.; Baran, P. S. Angew. Chem., Int. Ed. 2011, 50, 3362. (d) Liu, C.; Zhang, H.; Shi, W.; Lei, A. Chem. Rev. 2011, 111, 1780. (e) Engle, K. M.; Mei, T.-S.; Wasa, M.; Yu, J.-Q. Acc. Chem. Res. 2012, 45, 788. (f) Roizen, J. L.; Harvey, M. E. Du Bois, J. Acc. Chem. Res. 2012, 45, 911. (g) Rouquet, G.; Chatani, N. Angew. Chem., Int. Ed. 2013, 52, 11726. (h) He, J.; Wasa, M.; Chan, K. S. L.; Shao, Q.; Yu, J.-Q. Chem. Rev. 2017, 117, 8754. (i) Le Bras, J.; Muzart, J. Chem. Rev. 2011, 111, 1170. (j) Sun, C.-L.; Li, B.-J.; Shi, Z.-J. Chem. Rev. 2011, 111, 1293. (k) Cho, S. H.; Kim, J. Y.; Kwak, J.; Chang, S. Chem. Soc. Rev. 2011, 40, 5068. (1) Shang, X.; Liu, Z.-Q. Chem. Soc. Rev. 2013, 42, 3253; (m) Yang, L.; Huang, H. Chem. Rev. 2015, 115, 3468. (n) Guo, X.-X.; Gu, D.-W.; Wu, Z.; 
Zhang, W. Chem. Rev. 2015, 115, 1622. (o) Zheng, Q.-Z.; Jiao, N. Chem. Soc. Rev. 2016, 45, 4590. (p) Murakami, K.; Yamada, S.; Kaneda, T.; Itami, K. Chem. Rev. 2017, 117, 9302. (q) Yuan, S.; Wang, Y.; Qiu, G.; Liu, J. Chin. J. Org. Chem. 2017, 37, 566. (袁斯 甜, 王艳华, 邱观音生, 刘晋彪, 有机化学, 2017, 37, 566.) (r) Zhang, J. J.; Cheng, Y. B.; Duan, X. H. Chin. J. Chem. 2017, 35, 311. (s) Ruan, L.; Chen, C.; Zhang, X.; Sun, J. Chin. J. Org. Chem. 2018, 38, 3155. (阮利衡, 陈春欣, 张晓欣, 孙京, 有机化学, 2018, 38, 3155.) (t) Zhang, X.; Li, P.; Yuan, Y.; Jia, X. Chin. J. Org. Chem. 2018, 38, 2435. (张学文, 李鹏飞, 袁宇, 贾晓东, 有机化学, 2018, 38, 2435.) (u) Gu Z.; Ji S. Acta. Chim. Sinica 2018, 76, 347. (顾正 祥, 纪顺俊, 化学学报, 2018, 76, 347.)

[3] For selected recent reviews, see: (a) Shang, X.; Liu, Z.-Q. Acta Chim. Sinica 2015, 73, 1275. (尚䈗洁, 柳忠全, 化学学报, 2015, 73, 1275.) (b) Yi, H.; Zhang, G.; Wang, H.; Huang, Z.; Wang, J.; Singh, A. K.; Lei, A. Chem. Rev. 2017, 117, 9016.

[4] (a) Harris, E. F. P.; Waters, W. A. Nature 1952, 170, 212. (b) Walling, C. Pure Appl. Chem. 1967, 15, 69. (c) Tedder, J. M. Angew. Chem. Int. Ed. Engl. 1982, 21, 401. (d) Giese, B. Angew. Chem. Int.
Ed. Engl. 1989, 28, 969. (e) Roberts, B. P. Chem. Soc. Rev. 1999, 28 25 .

[5] Ravelli, D.; Fagnoni, M.; Fukuyama, T.; Nishikawa, T.; Ryu, I. ACS Catal. 2018, 8, 701.

[6] Tian, Y.; Sun, C.; Tan, R. X.; Liu, Z.-Q. Green Chem. 2018, 20, 588.

[7] (a) Snider, B. B. Chem. Rev. 1996, 96, 339. (b) Heiba, E. I.; Dessau, R. M. J. Am. Chem. Soc. 1971, 93, 524. (c) Iwahama, T.; Sakaguchi, S.; Ishii, Y. Chem. Commun. 2000, 2317. (d) Linker, U.; Kersten, B.; Linker, T. Tetrahedron 1995, 51, 9917. (e) Xie, J.; Huang, Z.-Z. Chem. Commun. 2010, 46, 1947. (f) Zhu, L.; Chen, H.; Wang, Z.; Li, C. Org. Chem. Front. 2014, 1, 1299. (g) Schweitzer-Chaput, B.; Demaerel, J.; Engler, H.; Klussmann, M. Angew. Chem., Int. Ed. 2014, 53, 8737. (h) Chu, X.; Meng, H.; Zi, Y.; Xu, X.-P.; Ji, S.-J. Chem.-Eur. J. 2014, 20, 17198. (i) Lan, X.; Wang, N.-X.; Zhang, W.; Wen, J.; Bai, C.; Xing, Y.-L.; Li, Y.-H. Org. Lett. 2015, 17, 4460. (j) Shiraishi, Y.; Tsukamoto, D.; Hirai, T. Org. Lett. 2008, 10, 3117. (k) Tsukamoto, D.; Shiraishi, Y.; Hirai, T. J. Org. Chem. 2010, 75, 1450.

[8] (a) Liu, Z.-Q.; Li, Z. Chem. Commun. 2016, 52, 14278. (b) Xu, Z.; Hang, Z.; Chai, L.; Liu, Z.-Q. Org. Lett. 2016, 18, 4662.

(Zhao, C.) 